\title{
Modal Analysis of In-Wheel Motor-Driven Electric Vehicle Based on Bond Graph Theory
}

\author{
Di Tan, Qiang Wang, and Yanshou Wu \\ School of Transportation and Vehicle Engineering, Shandong University of Technology, Zibo, China \\ Correspondence should be addressed to Di Tan; di.tan@mail.scut.edu.cn
}

Received 18 January 2017; Revised 26 March 2017; Accepted 12 April 2017; Published 18 July 2017

Academic Editor: Matteo Aureli

Copyright (C) 2017 Di Tan et al. This is an open access article distributed under the Creative Commons Attribution License, which permits unrestricted use, distribution, and reproduction in any medium, provided the original work is properly cited.

\begin{abstract}
A half-car vibration model of an electric vehicle driven by rear in-wheel motors was developed using bond graph theory and the modular modeling method. Based on the bond graph model, modal analysis was carried out to study the vibration characteristics of the electric vehicle. To verify the effectiveness of the established model, the results were compared to ones computed on the ground of modal analysis and Newton equations. The comparison shows that the vibration model of the electric vehicle based on bond graph theory not only is able to better compute the natural frequency but also can easily determine the deformation mode, momentum mode, and other isomorphism modes and describe the dynamic characteristics of an electric vehicle driven by in-wheel motors more comprehensively than other modal analysis methods.
\end{abstract}

\section{Introduction}

Vehicle vibration has been a hot spot in the field of automotive research. One method of studying vehicle vibration is modal analysis. Modal analysis of a vehicle vibration model allows us to observe the effects of varying vibration frequencies on vehicle vibration. The information gleaned from such analysis can aid further study of vehicle vibration characteristics. The chassis structures of in-wheel motors- (IWMs-) driven electric vehicles (EVs) and traditional centralized motordriven vehicles are significantly different. The IWM-driven EVs do not require a complex gearbox, clutch, differential mechanism, half-axis, and other transmission components, as the electric machine, reducer, brake, and other components are largely integrated into the wheel. Altering the chassis structure will inevitably result in novel vibration issues. Consequently, modal analysis must first be performed for the IWM-driven EVs.

At present, most vibration models and analyses of vehicles are based on Lagrangian or Newtonian theory $[1,2]$. At the end of the 1950s, Professor Paynter, of the United States, proposed bond graph (BG) theory, which was based on the theory of power flow transmission, conversion, storage, and dissipation. The theory elucidates a complete causal relationship and can clearly express the relationship between the mathematical model and the physical model. It is recognized as the most suitable modeling method of the state space equation, which is obtained from the domain of the physical system directly, and it is widely used in a number of disciplines [3-7]. A review of literature indicates that the BG method has been applied successfully to the modal analysis of a variety of mechanical systems. However, there have been relatively few studies regarding the application of BG theory to vehicle vibration $[8-10]$.

In this paper, BG theory and the modular modeling method are applied to the modal analysis of an IWM-driven EV. First, a half-car vibration model of a rear IWM-driven $\mathrm{EV}$ is established. Based on the physical vibration model, BG model is developed, and the mathematical model is deduced using BG theory. Afterward, modal analysis is carried out to study the vibration characteristics of the EV. Finally, modal analysis is conducted via the Newton method to verify the correctness and applicability of the established model.

\section{Vibration Model of the IWM-Driven EV}

2.1. Physical Model. This study considers a typical IWMdriven EV. The IWM is a permanent magnet synchronous 


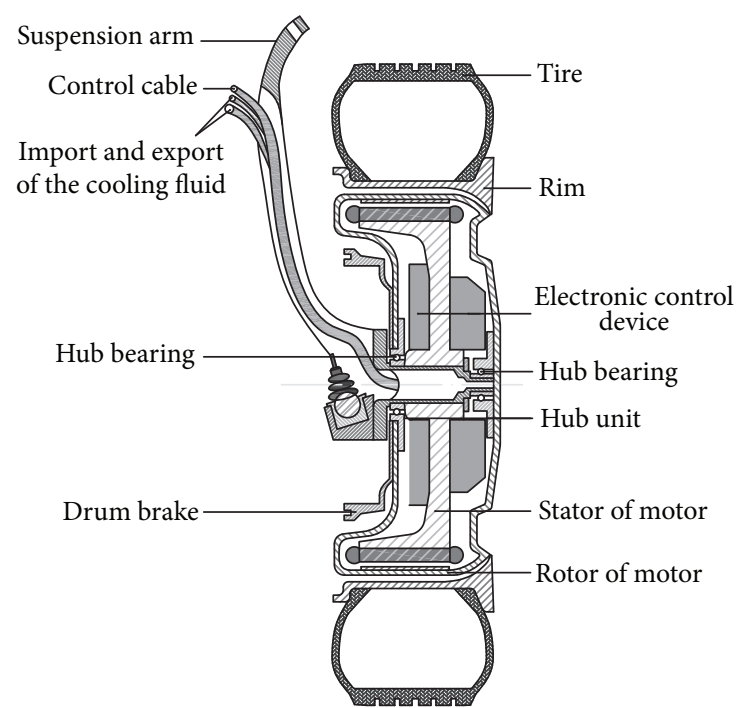

FIGURE 1: Structure of the IWM-driven system.

motor with an outer rotor and an inner stator. The structure of the IWM is shown in Figure 1 [11]. The IWM-driven system consists of the IWM, drum brake, hub bearing, wheel rim, tire, and so on. The IWM rotor is directly connected to the rim, and the vehicle can be driven by controlling the rotation of the IWM rotor. With this structure are two bearing structures, of which the hub bearing serves as a movable connecting unit and a load supporting unit; it is equivalent to a spring-damper system in the vertical direction.

Before developing the physical model, the following assumptions were made based on the structure of the IWMdriven EVs (Figure 1). (1) The car body is a rigid body, (2) the vehicle maintains a constant speed in a straight line, (3) the hub bearing is equivalent to a spring-damper system in the vertical direction, (4) the spring force of each component is a linear function of its displacement, while the damping force is a linear function of its speed, and (5) the car body only has 2 degrees of freedom, including vertical and pitch, while the unsprung mass has 3 degrees of freedom in the vertical direction.

Based on the above assumptions, the $1 / 2$ physical model of the IWM-driven EV is shown in Figure 2, where $m_{b}$ is the body mass, $I_{y}$ is the body pitching moment of inertia, $m_{f 1}$ is the unsprung mass of front wheel, $m_{r 11}$ is total mass of the rear wheel tires and rims, $m_{r 12}$ is the IWM rotor mass, $m_{r 21}$ is the mass of the support shaft and the brake, $m_{r 22}$ is the mass of the IWM stator, $l_{f}$ is the distance from the center of mass to the front axle, $l_{r}$ is the distance from the center of mass to the rear axle, $\theta$ is the body pitching angle, $k_{f 2}$ and $k_{f 1}$ are the stiffness of the front tire and the front suspension, respectively, $c_{f 2}$ and $c_{f 1}$ are the damping coefficients of the front tire and the front suspension, respectively, $k_{r 21}$ and $k_{r 22}$ are the bearing stiffness, $c_{r 21}$ and $c_{r 22}$ are the bearing damping coefficients, $k_{r 3}$ and $k_{r 1}$ are the stiffness of the rear tire and the rear suspension, respectively, $c_{r 3}$ and $c_{r 1}$ are the damping coefficients of the rear tire and the rear suspension, respectively, $z_{f 1}, z_{r 1}$, and $z_{r 2}$ are the vertical

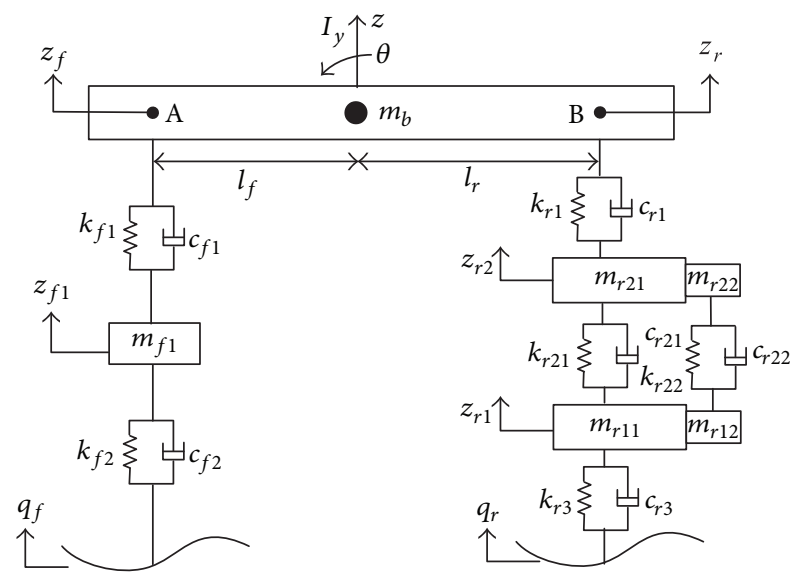

FIGURE 2: IWM-driven EV 1/2 physical model.

displacements of the corresponding mass blocks, $z_{f}$ and $z_{r}$ are the vertical displacements of points A and B, respectively, where $\mathrm{A}$ and $\mathrm{B}$ are the two connecting points of the car body and the suspensions, and $q_{f}$ and $q_{r}$ are the road displacement excitation of the front and rear wheel, respectively.

2.2. BG Model. For mechanical systems, the BG model consists of five basic components [3]. The $R$ component represents damping, the $C$ component represents spring, the I component represents the mass block or moment of inertia, the Se component represents the power source, and the $S_{f}$ component represents the source of the speed. Furthermore, the $R$ component is the energy dissipation element, and the $C$ and $I$ components are energy storage elements. The five elements are linked by constant velocity 1 junctions, constant force 0 junctions, transformer TF, and so on.

Development of the BG model proceeds as follows. Considering the structural characteristics of the IWM-driven EV and the advantages of the BG method, the physical model shown in Figure 2 is divided into three submodules according to the modular modeling method. This includes the car body module and the front and rear suspension modules. Next, the BG model of the three submodules is developed using BG theory as shown in Figure 3. Finally, complete BG model of the EV can be obtained by composing the three submodules as shown in Figure 4.

From the fact that ports A and B in Figures 3(a) and 3(b) are connected with corresponding ports $\mathrm{A}$ and $\mathrm{B}$ in Figure 3(c), the complete BG model of the 1/2 IWM-driven EV can be obtained as shown in Figure 4.

Based on the modeling process described above, it can be observed that modular modeling based on BG theory simplifies the otherwise complex model. If the resulting model needs to be corrected, only the necessary submodule must be modified, which does not affect the structures of the other submodules.

2.3. Mathematical Model. In BG theory, the symbols $q$ and $p$ represent the displacement and momentum, respectively, in a translational motion, whereas they represent the angle 

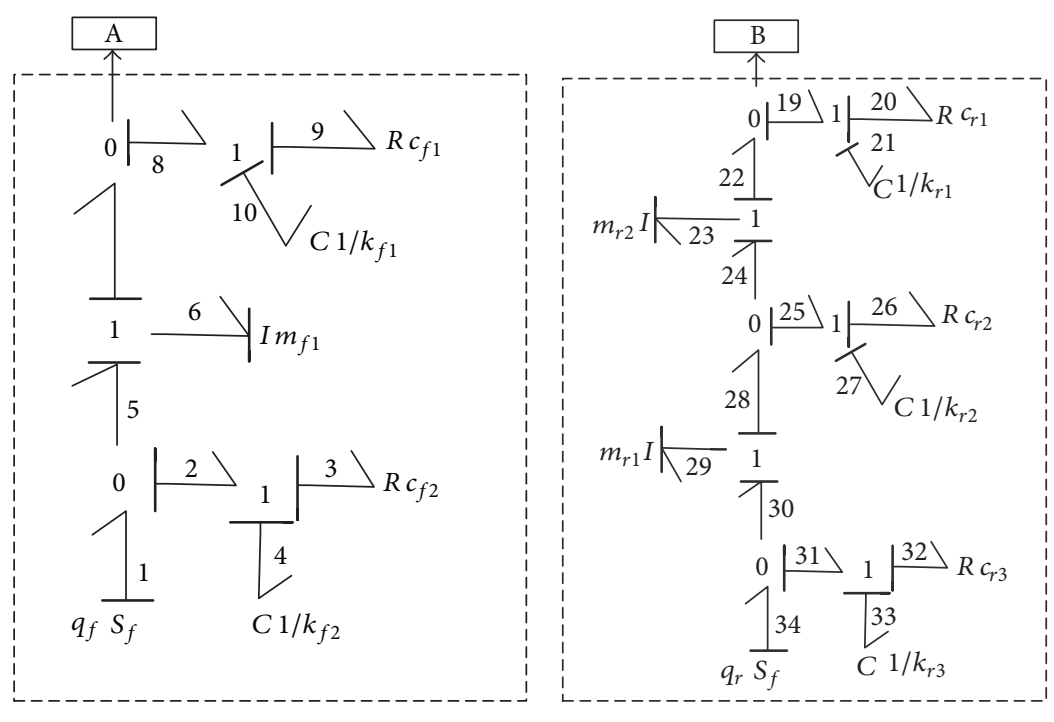

(a) BG model of the front suspension module

(b) BG model of the rear suspension module

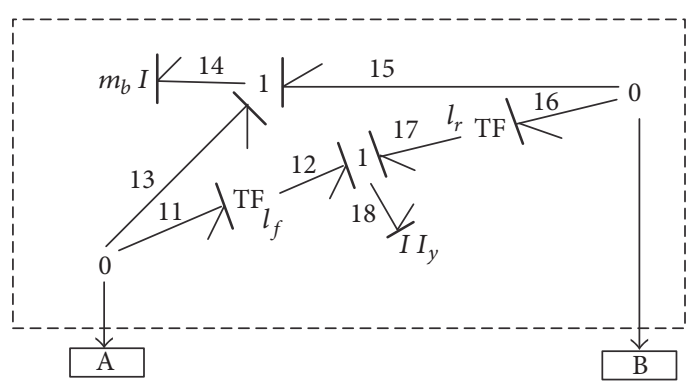

(c) BG model of the car body module

FIGURE 3: BG model of three submodules.

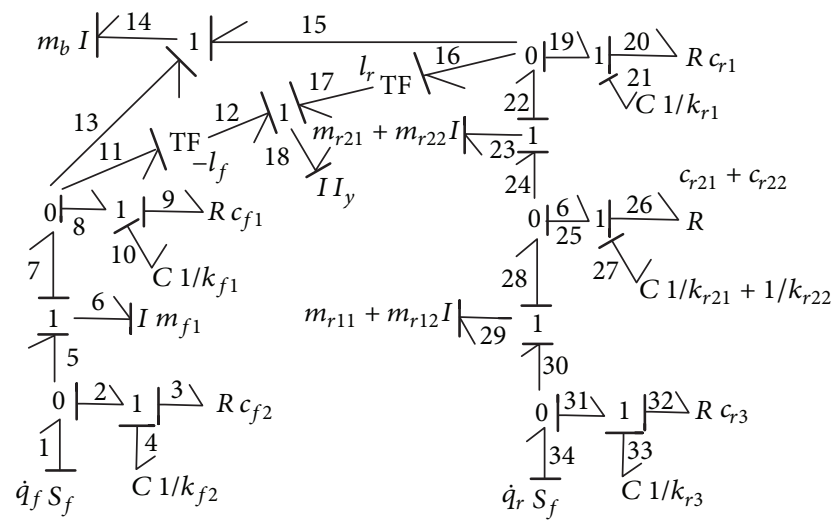

Figure 4: BG model of the $1 / 2$ vehicle.

and angular momentum in a rotational motion. The symbols $\dot{q}$ and $\dot{p}$ represent the velocity and force in a translational motion and represent angular velocity and torque in a rotational motion. Each element in the BG model has a serial number corresponding to the different elements. Here, $\dot{q}_{i}$ $(i=f, r)$ refers to the road surface roughness (RSR) velocity excitation, and in the other elements with serial numbers, $q$, $p, \dot{q}$, and $\dot{p}$ refer to the corresponding symbols in the physical model.

Based on the BG model developed in Figure 4, the mathematical model of the vibration model can be deduced as follows using the causality and power flow direction of BG theory.

The vertical vibration velocity equation of $m_{f 1}$ is

$$
\dot{q}_{4}=\dot{q}_{f}-\frac{p_{6}}{m_{f 1}} .
$$
is

The vertical vibration velocity equation of $m_{r 11}$ and $m_{r 12}$

$$
\dot{q}_{33}=\dot{q}_{r}-\frac{p_{29}}{\left(m_{r 11}+m_{r 12}\right)} .
$$
is

The vertical vibration velocity equation of $m_{r 21}$ and $m_{r 22}$

$$
\dot{q}_{27}=\frac{p_{29}}{\left(m_{r 11}+m_{r 12}\right)}-\frac{p_{23}}{\left(m_{r 21}+m_{r 22}\right)} .
$$


The vertical vibration velocity equations of the two endpoints $\mathrm{A}$ and $\mathrm{B}$ at the interface between the car body and suspensions are

$$
\begin{aligned}
& \dot{q}_{10}=\frac{p_{6}}{m_{f 1}}+l_{f} \frac{p_{18}}{I_{y}}-\frac{p_{14}}{m_{b}} \\
& \dot{q}_{21}=\frac{p_{23}}{\left(m_{r 21}+m_{r 22}\right)}-l_{r} \frac{p_{18}}{I_{y}}-\frac{p_{14}}{m_{b}} .
\end{aligned}
$$

The vertical force balance equation of $m_{f 1}$ is

$$
\dot{p}_{6}=c_{f 2} \dot{q}_{4}+k_{f 2} q_{4}-c_{f 1} \dot{q}_{10}-k_{f 1} q_{10}
$$

The vertical force balance equation of $m_{r 1}$ is

$$
\begin{aligned}
\dot{p}_{29}= & c_{r 3} \dot{q}_{33}+k_{r 3} q_{33}-\left(c_{r 21}+c_{r 22}\right) \dot{q}_{27} \\
& -\left(k_{r 21}+k_{r 22}\right) q_{27} .
\end{aligned}
$$

The vertical force balance equation of $m_{r 2}$ is

$$
\begin{aligned}
\dot{p}_{23}= & \left(c_{r 21}+c_{r 22}\right) \dot{q}_{27}+\left(k_{r 21}+k_{r 22}\right) q_{27}-c_{r 1} \dot{q}_{21} \\
& -k_{r 1} q_{21} .
\end{aligned}
$$

The vertical force balance equation of the car body $m_{b}$ is

$$
\dot{p}_{14}=c_{r 1} \dot{q}_{21}+k_{r 1} q_{21}+c_{f 1} \dot{q}_{10}+k_{f 1} q_{10} .
$$

The pitching moment equation of the car body $m_{b}$ is

$$
\dot{p}_{18}=-l_{f}\left(c_{f 1} \dot{q}_{10}+k_{f 1} q_{10}\right)+l_{r}\left(c_{r 1} \dot{q}_{21}+k_{r 1} q_{21}\right) .
$$
follows:

Equations (1)-(9) can be expressed in matrix form as

$$
\dot{X}=A X+B \dot{X}+U
$$

where

$$
\begin{aligned}
& \dot{X}=\left[\begin{array}{llllllllll}
\dot{q}_{4} & \dot{q}_{33} & \dot{q}_{27} & \dot{q}_{10} & \dot{q}_{21} & \dot{p}_{6} & \dot{p}_{29} & \dot{p}_{23} & \dot{p}_{14} & \dot{p}_{18}
\end{array}\right]^{T} ; \\
& X=\left[\begin{array}{llllllllll}
q_{4} & q_{33} & q_{27} & q_{10} & q_{21} & p_{6} & p_{29} & p_{23} & p_{14} & p_{18}
\end{array}\right]^{T} \text {; } \\
& k_{r 2}=k_{r 21}+k_{r 22} \text {; } \\
& m_{r 1}=m_{r 11}+m_{r 12} \\
& m_{r 2}=m_{r 21}+m_{r 22} \\
& U=\left[\begin{array}{llllllllll}
\dot{q}_{f} & \dot{q}_{r} & 0 & 0 & 0 & 0 & 0 & 0 & 0 & 0
\end{array}\right]^{T} ; \\
& A=\left[\begin{array}{cc}
0 & A_{q} \\
A_{p} & 0
\end{array}\right] \text {; }
\end{aligned}
$$

$$
\begin{aligned}
& B=\left[\begin{array}{cc}
0 & 0 \\
B_{p} & 0
\end{array}\right] ; \\
& A_{p}=\left[\begin{array}{ccccc}
k_{f 2} & 0 & 0 & -k_{f 1} & 0 \\
0 & k_{r 3} & -k_{r 2} & 0 & 0 \\
0 & 0 & k_{r 2} & 0 & -k_{r 1} \\
0 & 0 & 0 & k_{f 1} & k_{r 1} \\
0 & 0 & 0 & -l_{f} k_{f 1} & l_{r} k_{r 1}
\end{array}\right] ;
\end{aligned}
$$$$
A_{q}=\left[\begin{array}{ccccc}
-\frac{1}{m_{f 1}} & 0 & 0 & 0 & 0 \\
0 & -\frac{1}{m_{r 1}} & 0 & 0 & 0 \\
0 & \frac{1}{m_{r 1}} & -\frac{1}{m_{r 2}} & 0 & 0 \\
\frac{1}{m_{f 1}} & 0 & 0 & -\frac{1}{m_{b}} & \frac{l_{f}}{I_{y}} \\
0 & 0 & \frac{1}{m_{r 2}} & -\frac{1}{m_{b}} & -\frac{l_{r}}{I_{y}}
\end{array}\right]
$$

$$
B_{p}=\left[\begin{array}{ccccc}
c_{f 2} & 0 & 0 & -c_{f 1} & 0 \\
0 & c_{r 3} & -c_{r 2} & 0 & 0 \\
0 & 0 & c_{r 2} & 0 & -c_{r 1} \\
0 & 0 & 0 & c_{f 1} & c_{r 1} \\
0 & 0 & 0 & -l_{f} c_{f 1} & l_{r} c_{r 1}
\end{array}\right] \text {. }
$$

\section{Vibration Modal Analysis of the IWM- Driven Vehicle Based on BG Theory}

3.1. Basic Theory of BG Modal Analysis. According to (10), the undamped free vibration equation of the vehicle can be deduced as follows:

$$
\dot{X}=A X
$$

The eigenvalue and eigenvector of the vibration system can be obtained from the following equation:

$$
A \varphi_{i}=\lambda \varphi_{i}
$$

where $\varphi_{i}$ is the eigenvector and $\lambda$ is the eigenvalue.

According to (13), dual modes $\varphi_{q}$ (deformation modes) and $\varphi_{p}$ (momentum modes) can be obtained directly. In addition, isomorphic modes, such as the displacement modes, velocity modes, and force modes, can be obtained easily through a simple formula transformation.

3.2. Modal Analysis Based on BG Theory. The basic parameters of the IWM-driven EV studied in this paper are shown in Table 1.

Substituting the parameters in Table 1 into (10), the natural frequency and vibration mode of the vehicle can be calculated. This was achieved by a program written in MATLAB. The results are shown in Table 2. 
TABLE 1: Vehicle parameters.

\begin{tabular}{lcc}
\hline Variable & Numerical value & Unit \\
\hline$m_{b}$ & 790 & $\mathrm{~kg}$ \\
$k_{f 1} / k_{r 1}$ & $80 / 80$ & $\mathrm{kN} / \mathrm{m}$ \\
$k_{f 2} / k_{r 3}$ & $400 / 400$ & $\mathrm{kN} / \mathrm{m}$ \\
$k_{r 21} / k_{r 22}$ & $5000 / 5000$ & $\mathrm{kN} / \mathrm{m}$ \\
$c_{f 1} / c_{r 1}$ & $5000 / 5000$ & $\mathrm{~N} \cdot \mathrm{s} / \mathrm{m}$ \\
$c_{f 2} / c_{r 3}$ & $100 / 100$ & $\mathrm{~N} \cdot \mathrm{s} / \mathrm{m}$ \\
$c_{r 21} / c_{r 22}$ & $0 / 0$ & $\mathrm{~N} \cdot \mathrm{s} / \mathrm{m}$ \\
$m_{f 1}$ & 60 & $\mathrm{Kg}$ \\
$m_{r 11} / m_{r 12}$ & $50 / 30$ & $\mathrm{Kg}$ \\
$m_{r 21} / m_{r 22}$ & $20 / 30$ & $\mathrm{Kg}$ \\
$I_{y}$ & 1300 & $\mathrm{~kg} \cdot \mathrm{m}^{2}$ \\
$l_{f} / l_{r}$ & $1.2 / 1.4$ & $\mathrm{~m}$ \\
\hline
\end{tabular}

As seen in Table 2, the deformation mode $\varphi_{q}$ and the momentum mode $\varphi_{p}$ can be obtained from formula (12). Mode $\varphi_{q}$, corresponding to the state variable $q$, is the deformation mode and the orderliness formed by the capacitive element. Mode $\varphi_{p}$, corresponding to the state variable $p$, expresses the momentum of the inertial element in the system.

The deformation of each capacitive element at a certain frequency can be determined from the deformation mode $\varphi_{q}$. Among these, $q_{4}$ and $q_{33}$ are the deformations of the front and rear tires, respectively, related to the grounding safety of the vehicle. $q_{10}$ and $q_{21}$ are the deformations of the front and rear suspensions, respectively, related to the riding comfort of the vehicle. $q_{27}$ is the deformation of the hub bearing, that is, the relative displacement between the IWM stator and the rotor, which is important for the running safety of the IWM. Therefore, the analysis of the deformation mode $\varphi_{q}$ is of great value in determining the vehicle's vibration characteristics.

To better present the deformation mode clearly, the data obtained from the above table were normalized, and the diagram of the deformation mode of the capacitive elements is shown in Figure 5.

As seen from Figure 5, (1) when the excited frequency $\omega$ is close to $1.9972 \mathrm{~Hz}$, the deformation of the front suspension is greatest, and the deformation of the other capacitive elements is relatively small; (2) when the excited frequency $\omega$ is close to $2.1462 \mathrm{~Hz}$, the deformation of the rear suspension is greatest, and the deformation of the other capacitive elements is relatively small; (3) when the excited frequency $\omega$ is close to the third-order natural frequency of $9.7115 \mathrm{~Hz}$, the deformations of both the rear suspension and the rear tire are larger, while the deformations of the other capacitive elements are relatively small. At $9.7115 \mathrm{~Hz}$, the deformation primarily occurs in the rear axle; (4) when the excited frequency $\omega$ is close to the fourth-order natural frequency of $14.2639 \mathrm{~Hz}$, the deformations of both the front suspension and the front tire are relatively larger, while the deformations of the other capacitive elements are relatively small. At this frequency, the deformation primarily occurs in the rear axle; (5) when the excited frequency $\omega$ is close to the fifthorder natural frequency of $91.1385 \mathrm{~Hz}$, the deformation of the hub bearing is greatest, followed by those of the rear suspension and the rear tire, while the deformations of the front suspension and the front tire are relatively small. From this, it can be observed that a high excited frequency can cause large deformations in the bearing, which results in a big relative displacement between the IWM stator and rotor. A large relative displacement will cause large electromagnetic force excitation, and if the relative displacement exceeds the safety limit, the vehicle will be in danger. The deformation modes can also be translated into the force mode, strain mode, and stress mode, depending on the purpose of the analysis.

In addition, the velocity vibration mode of the inertial components is an issue of general concern in vehicle vibration and is important for the evaluation of vehicle ride comfort. The vibration velocity mode can be obtained by the following transformation formula:

$$
\varphi_{v r}=\operatorname{diag}\left[I_{r}^{-1}\right] \varphi_{p r},
$$

where $I$ represents the inertia component corresponding to $r$ and $r=1,2, \ldots, 5$.

The data obtained from equation (14) are normalized, and the diagrams of the velocity vibration mode are shown in Figure 6.

As can be observed in Figure 6(a), when the excited frequency $\omega$ is close to the first-order natural frequency of $1.9972 \mathrm{~Hz}$, the vertical vibration velocity of the body mass is greatest, followed by those of the body pitching angle and the unsprung mass of the front wheel, while the vibration velocities of the rear IWM rotor and the rear wheel IWM stator are relatively small. Therefore, at $1.9972 \mathrm{~Hz}$, the vibration arises primarily from the car body, but the vertical and pitching angle velocities of the car body vibrate in opposite directions.

As can be observed in Figure 6(b), when the excited frequency $\omega$ is close to the second-order natural frequency of $2.1462 \mathrm{~Hz}$, the pitch angle velocity and the vertical vibration velocity of the car body are both relatively large, followed by the vertical vibration velocity of the IWM stator and rotor in the rear wheel. The vertical velocity of the unsprung mass of the front wheel is at a minimum. Therefore, vibration arises mainly from the car body at this frequency, and the vertical and pitching angle velocities of the car body vibrate in the same direction.

As can be observed in Figure 6(c), when the excited frequency $\omega$ is close to the third-order natural frequency of $9.7115 \mathrm{~Hz}$, the vertical vibration velocities of the IWM stator and rotor in the rear wheel are relatively large, whereas the vertical vibration velocity of the front wheel unsprung mass and the vertical vibration velocity and pitch angle velocity of the car body are relatively small. Therefore, the vibration is primarily a result of the IWM at this frequency, and the vertical vibration velocities of the IWM stator and rotor vibrate in the same direction.

As can be observed in Figure 6(d), when the excited frequency $\omega$ is close to the fourth-order natural frequency of 
TABLE 2: Natural frequencies and vibration modes.

\begin{tabular}{|c|c|c|c|c|c|c|}
\hline Natural frequency & & $\begin{array}{c}\lambda_{1} \\
1.9972 \\
\end{array}$ & $\begin{array}{c}\lambda_{2} \\
2.1462 \\
\end{array}$ & $\begin{array}{c}\lambda_{3} \\
9.7115\end{array}$ & $\begin{array}{c}\lambda_{4} \\
14.2639\end{array}$ & $\begin{array}{c}\lambda_{5} \\
91.1385 \\
\end{array}$ \\
\hline \multirow{5}{*}{ Vibration mode } & \multirow{5}{*}{$\varphi_{q}$} & 0.0021 & -0.0001 & 0 & 0.018 & 0 \\
\hline & & 0.0002 & 0.002 & 0.016 & 0 & 0.0016 \\
\hline & & 0 & 0.0001 & 0.0002 & 0 & -0.0040 \\
\hline & & 0.0102 & -0.0007 & 0 & -0.0184 & 0 \\
\hline & & 0.0007 & 0.0096 & -0.0172 & 0 & 0.0025 \\
\hline \multirow{5}{*}{ Vibration mode } & \multirow{5}{*}{$\varphi_{p}$} & 1.5751 & -0.1154 & 0.0115 & 96.6492 & 0 \\
\hline & & 0.1533 & 2.2071 & 77.9663 & -0.0052 & 71.0787 \\
\hline & & 0.0995 & 1.4326 & 49.2266 & -0.0032 & -70.3383 \\
\hline & & 69.7208 & 52.9287 & -22.4850 & -16.4268 & 0.3434 \\
\hline & & -71.6693 & 84.8034 & -31.5027 & 19.7254 & 0.4808 \\
\hline
\end{tabular}

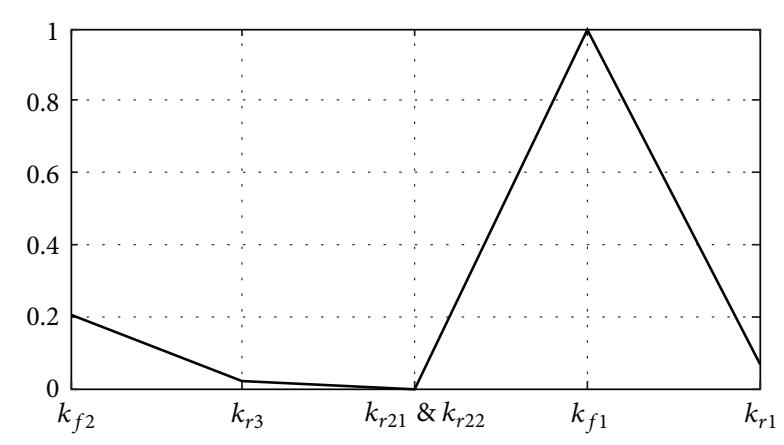

(a) First-order deformation mode $(1.9972 \mathrm{~Hz})$

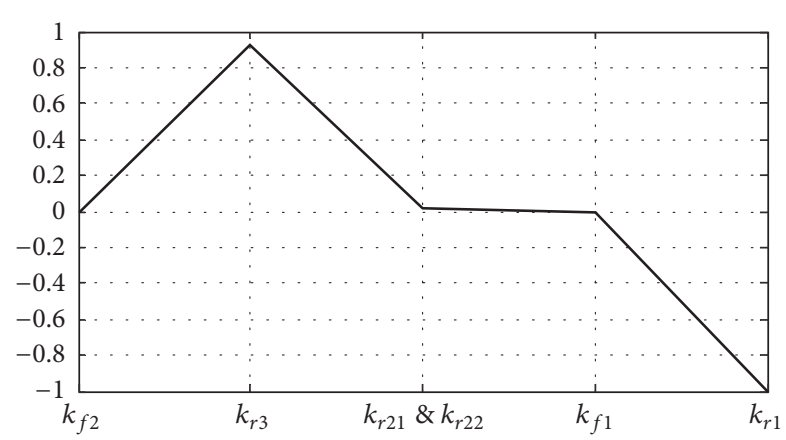

(c) Third-order deformation mode $(9.7115 \mathrm{~Hz})$

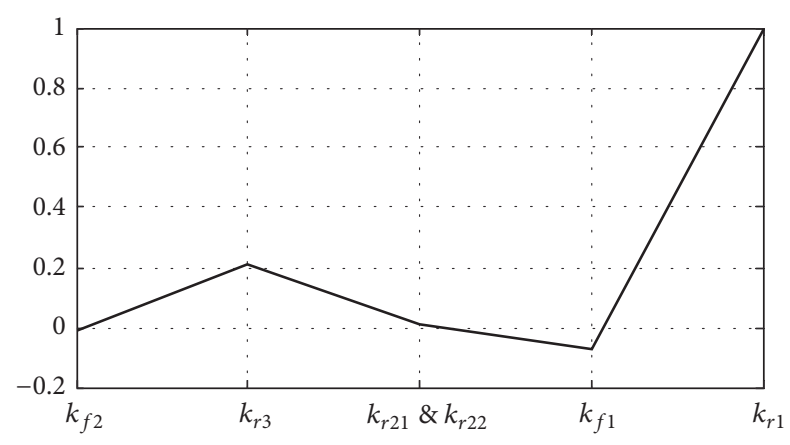

(b) Second-order deformation mode $(2.1462 \mathrm{~Hz})$

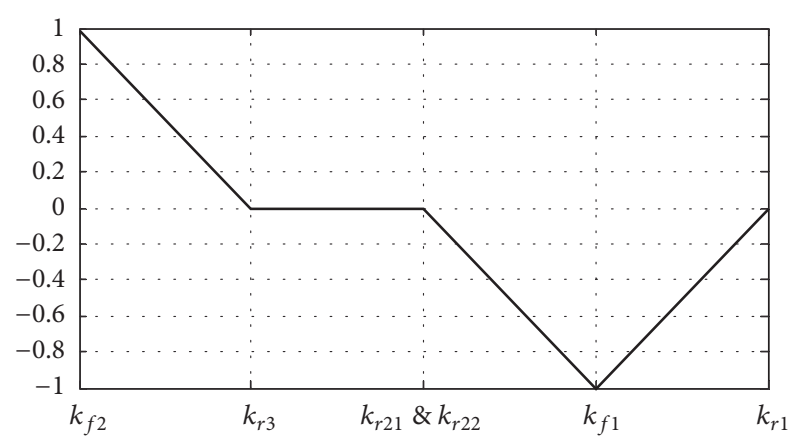

(d) Fourth-order deformation mode $(14.2639 \mathrm{~Hz})$

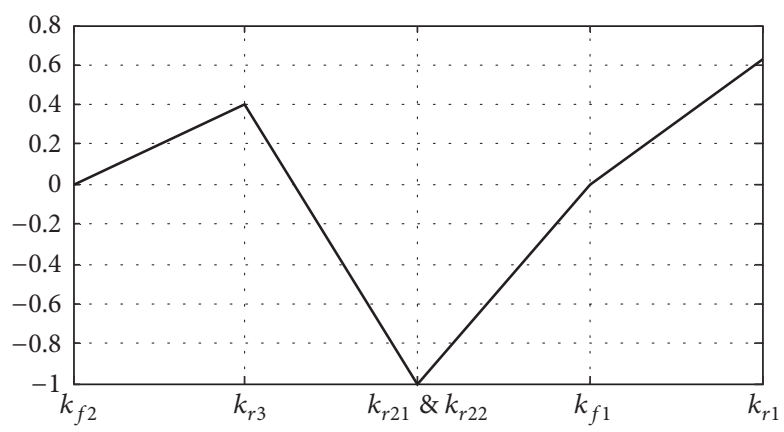

(e) Fifth-order deformation mode $(91.1385 \mathrm{~Hz})$

FIgURE 5: Vibration mode diagram of the deformation. 


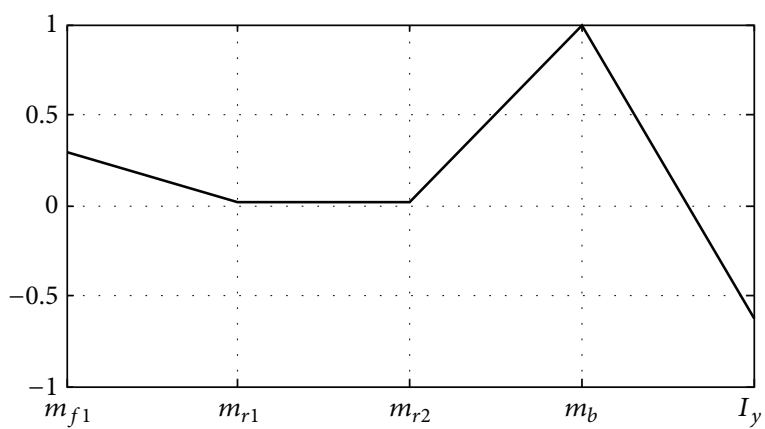

(a) First-order velocity vibration mode $(1.9972 \mathrm{~Hz})$

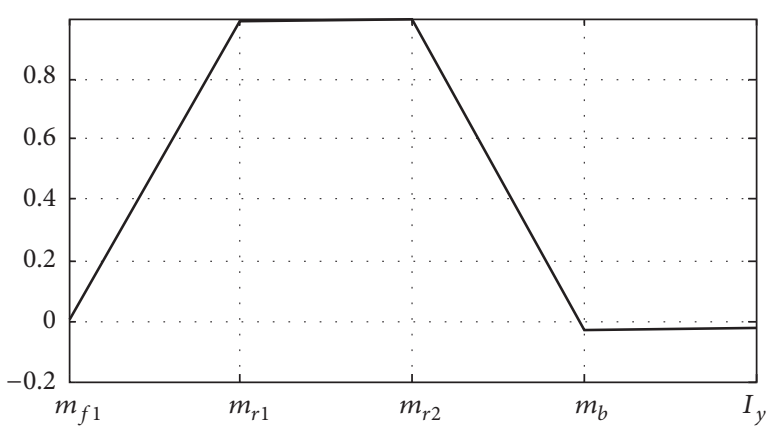

(c) Third-order velocity vibration mode $(9.7115 \mathrm{~Hz})$

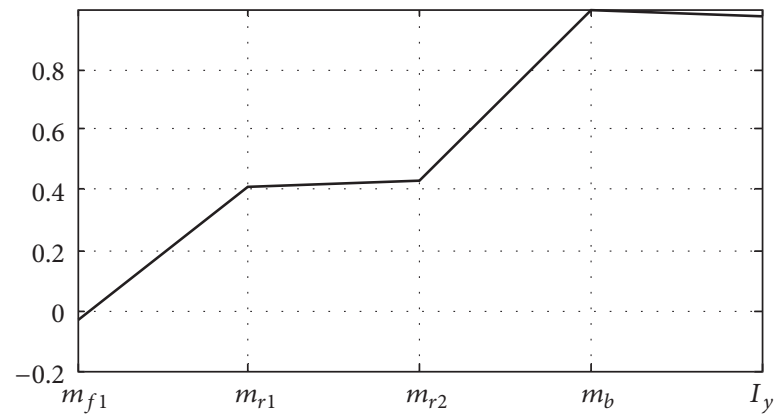

(b) Second-order velocity vibration mode $(2.1462 \mathrm{~Hz})$

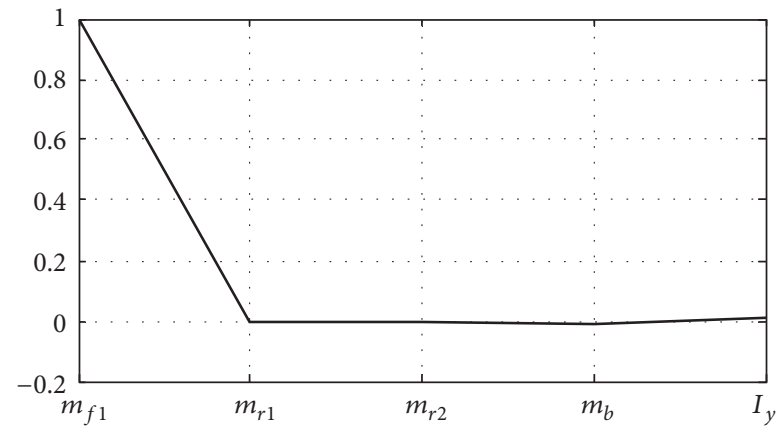

(d) Fourth-order velocity vibration mode $(14.2639 \mathrm{~Hz})$

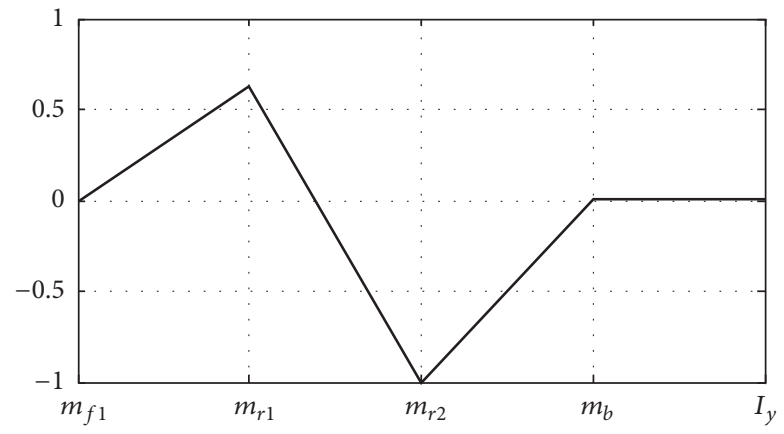

(e) Fifth-order velocity vibration mode $(91.1385 \mathrm{~Hz})$

FIGURE 6: Vibration mode diagram of the velocity.

14.2639 Hz, the vertical vibration velocity of the front wheel unsprung mass is greatest, and the vibration velocities of the other components are relatively small. At this frequency, the vertical vibration is primarily result of the front wheel unsprung mass.

As seen from Figure 6(e), when the excited frequency $\omega$ is close to the fifth-order natural frequency of $91.1385 \mathrm{~Hz}$, the vertical vibration velocity of the stator in rear wheel is greatest, followed by the vertical vibration velocity of the rotor. The vibration velocity of the other components is relatively small. Therefore, the vertical vibrations of the stator and rotor in rear wheel are the primary components of vibration, but they are moving in opposite directions.

From the above modal analysis result, it can be observed that the vibration model of the EV based on BG theory not only can calculate the natural frequency but also is able to obtain two modes directly, that is, the deformation mode and momentum mode. In addition, the other isomorphic modes can be obtained from the simple formula transformation. Modal analysis of the IWM-driven EV based on BG theory can comprehensively describe the dynamic characteristics.

\section{Contrast and Verification with the Newton Method}

4.1. Mathematical Model. Using Newton's Second Law, the mathematical model of the IWM-driven EV in Figure 2 can be obtained as follows.

The vertical and pitching equations of motion of the car body are

$$
\begin{aligned}
m_{b} \ddot{z}_{b}= & c_{f 1}\left(\dot{z}_{f 1}-\dot{z}_{f}\right)+k_{f 1}\left(z_{f 1}-z_{f}\right) \\
& +c_{r 1}\left(\dot{z}_{r 2}-\dot{z}_{r}\right)+k_{r 1}\left(z_{r 2}-z_{r}\right),
\end{aligned}
$$




$$
\begin{aligned}
I_{y} \ddot{\theta}= & -l_{f}\left[c_{f 1}\left(\dot{z}_{f 1}-\dot{z}_{f}\right)+k_{f 1}\left(z_{f 1}-z_{f}\right)\right] \\
& +l_{r}\left[c_{r 1}\left(\dot{z}_{r 2}-\dot{z}_{r}\right)+k_{r 1}\left(z_{r 2}-z_{r}\right)\right] .
\end{aligned}
$$

The vertical equations of motion of the unsprung mass are

$$
\begin{aligned}
m_{f 1} \ddot{z}_{f 1}= & c_{f 2}\left(\dot{q}_{f}-\dot{z}_{f 1}\right)+k_{f 2}\left(q_{f}-z_{f 1}\right) \\
& -c_{f 1}\left(\dot{z}_{f 1}-\dot{z}_{f}\right) \\
& -k_{f 1}\left(z_{f 1}-z_{f}\right), \\
\left(m_{r 11}+m_{r 12}\right) \ddot{z}_{r 1}= & c_{r 3}\left(\dot{q}_{r}-\dot{z}_{r 1}\right)+k_{r 3}\left(q_{r}-z_{r 1}\right) \\
& -\left(c_{r 21}+c_{r 22}\right)\left(\dot{z}_{r 1}-\dot{z}_{r 2}\right) \\
& -\left(k_{r 21}+k_{r 22}\right)\left(z_{r 1}-z_{r 2}\right),
\end{aligned}
$$

$$
\begin{aligned}
& \ddot{Y}=\left[\begin{array}{lllll}
\dot{z}_{f 1} & \dot{z}_{r 1} & \dot{z}_{r 2} & \dot{z}_{b} & \dot{\theta}
\end{array}\right]^{T} ; \\
& Y=\left[\begin{array}{lllll}
z_{f 1} & z_{r 1} & z_{r 2} & z_{b} & \theta
\end{array}\right]^{T} ;
\end{aligned}
$$$$
M=\left[\begin{array}{ccccc}
m_{f 1} & 0 & 0 & 0 & 0 \\
0 & m_{r 1} & 0 & 0 & 0 \\
0 & 0 & m_{r 2} & 0 & 0 \\
0 & 0 & 0 & m_{b} & 0 \\
0 & 0 & 0 & 0 & I_{y}
\end{array}\right]
$$$$
K=\left[\begin{array}{ccccc}
k_{f 1}+k_{f 2} & 0 & 0 & -k_{f 1} & l_{f} k_{f 1} \\
0 & k_{r 2}+k_{r 3} & -k_{r 2} & 0 & 0 \\
0 & -k_{r 2} & k_{r 1}+k_{r 2} & -k_{r 1} & -l_{r} k_{r 1} \\
-k_{f 1} & 0 & -k_{r 1} & k_{f 1}+k_{r 1} & l_{r} k_{r 1}-l_{f} k_{f 1} \\
l_{f} k_{f 1} & 0 & -l_{r} k_{r 1} & l_{r} k_{r 1}-l_{f} k_{f 1} & l_{f}^{2} k_{f 1}+l_{r}^{2} k_{r 1}
\end{array}\right] .
$$

4.2. Modal Analysis. Based on (15) and (16), the undamped free vibration equation can be expressed as follows:

$$
M \ddot{Y}+K Y=0,
$$

where

The natural frequencies of the system are obtained by a program written in MATLAB: $T_{1}=1.9972, T_{2}=2.1462, T_{3}=$ $9.7115, T_{4}=14.2639$, and $T_{5}=91.1385$, which is the same result calculated by the BG method.

Equation (17) shows that only five vibration modes of velocity can be obtained. The normalized result is identical to the result calculated by the BG method. These five vibration modes are not tabulated here.

The aforementioned comparison not only verifies the correctness of the mathematical model derived from the BG method but also shows the advantages of BG theory in modal analysis, which allows us to obtain the deformation modes and momentum modes and convert them into other desired modes. In addition, the Newton method for solving other modes, such as the displacement mode and the stress mode, requires the use of different formulas and cannot be easily transformed into other modes. This is one of the reasons why BG theory is widely applied in complex mechanical, electrical, fluid, and the other multienergy domain systems.

\section{Conclusion}

In this paper, BG theory and the modular modeling method are applied to vibration analysis of the IWM-driven EV. The effectiveness of the modeling method in this paper is verified by using the Newton method. Through the research presented in this paper, some useful conclusions can be formulated as follows.

(1) The modal analysis results for the IWM-driven EVs based on BG theory show that (1) for capacitive components in the system, a low excitation frequency primarily affects deformation of the suspension and the tire, and the high excitation frequency significantly affects not only the deformation of the hub bearing but also that of the rear 
suspension and tire; (2) for the inertia component, the firstand second-order frequencies primarily impact the vibration of the car body, whereas the third- and fifth-order frequencies primarily affect the vibration of the IWM stator and rotor components. The fourth-order frequency mainly affects the unsprung mass of the front axle.

(2) As known from the above results the high excitation frequency has significant effect on the IWM, which maybe causes the structure change of IWM and should be regarded with some care.

(3) Modal analysis based on BG theory is able to obtain more complete modal information to describe the dynamic behavior of the vehicle, while modular modeling based on BG theory simplifies the otherwise complex model, which facilitates subsequent modification.

The work in this study provides an idea and a method for vehicle vibration research and lays a foundation for further research on the vibration characteristics of IWM-driven EVs.

\section{Conflicts of Interest}

The authors declare that there are no conflicts of interest regarding the publication of this paper.

\section{Acknowledgments}

This research is supported by the National Natural Science Foundation of China (Grant no. 51405273) and sponsored by Shandong Province Higher Educational Science and Technology Program (Grant no. J14LB08).

\section{References}

[1] S. Timoshenko, S. H. Young, and W. Weaver, Vibration Problems in Engineering, John Wiley \& Sons, 4th edition, 1974.

[2] M. A. Eltantawie, "Decentralized neuro-fuzzy control for half car with semi-active suspension system," International Journal of Automotive Technology, vol. 13, no. 3, pp. 423-431, 2012.

[3] D. C. Karnopp, D. L. Margolis, and R. C. Rosenberg, System Dynamics: Modeling and Simulaion of Mechatronic Systems, John Wiley \& Sons, New York, NY, USA, 2006.

[4] H. M. Paynter, Analysis and Design of Engineering Systems, M.I.T. Press, Cambridge, Mass, USA, 1961.

[5] Troch, Borutzky, and P. Gawthrop, Mathematical \& Computer Modelling of Dynamical Systems, vol. 12, Taylor \& Francis, 2006.

[6] W. Borutzky, "Bond graphs and object-oriented modelling-a comparison," Proceedings of the Institution of Mechanical Engineers. Part I: Journal of Systems and Control Engineering, vol. 216, no. 1, pp. 25-33, 2002.

[7] R. Loureiro, R. Merzouki, and B. O. Bouamama, "Bond graph model based on structural diagnosability and recoverability analysis: Application to intelligent autonomous vehicles," Vehicular Technology, vol. 61, no. 3, pp. 986-997, 2012.

[8] W. Borutzky, B. Barnard, and J. U. Thoma, "Describing bond graph models of hydraulic components in Modelica," Mathematics and Computers in Simulation, vol. 53, no. 4-6, pp. 381$387,2000$.

[9] Orlikowski C. and R. Hein, "Modelling and analysis of beam/ bar structure by application of bond graphs," Journal of Theoretical and Applied Mechanics, vol. 49, no. 4, pp. 1003-1017, 2011.
[10] Z. Y. Zhao and Z. L. Bing, "Modal analysis and application research based on bond graph," in Machine Research and Application, vol. 3, pp. 7-10, 2014.

[11] D. Tan, Dynamic Characteristics and Structural Optimization of The Hub Motor Drive System with Built-In Mount, China University of Technology, Guangzhou, China, 2013. 


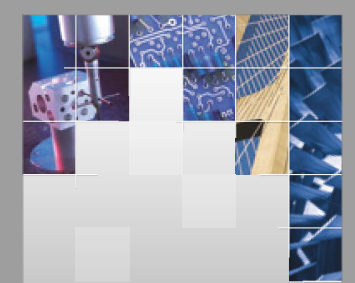

\section{Enfincering}
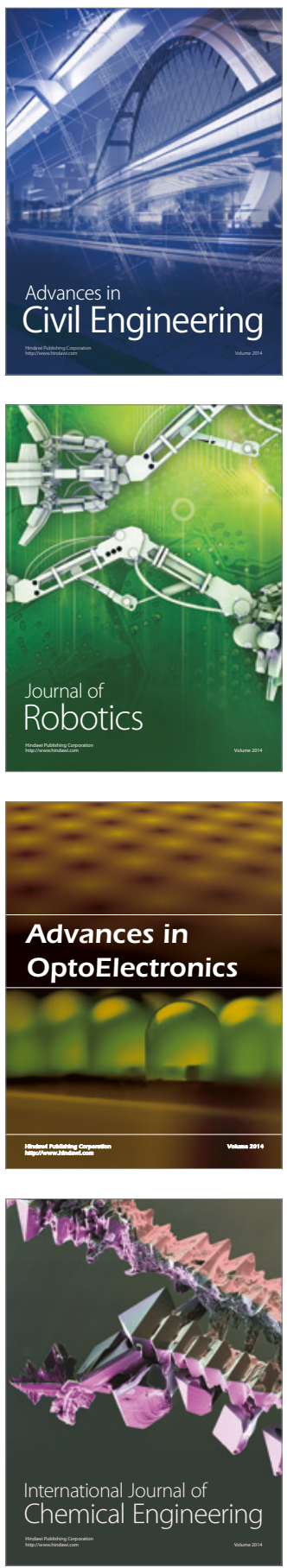

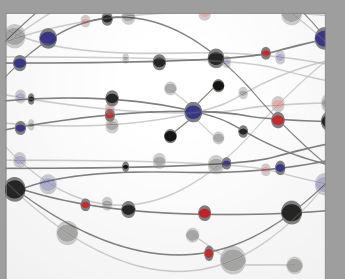

The Scientific World Journal

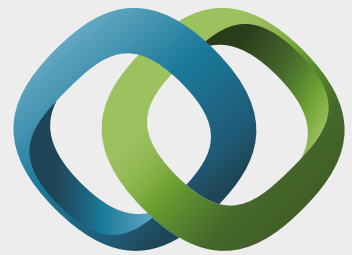

\section{Hindawi}

Submit your manuscripts at

https://www.hindawi.com
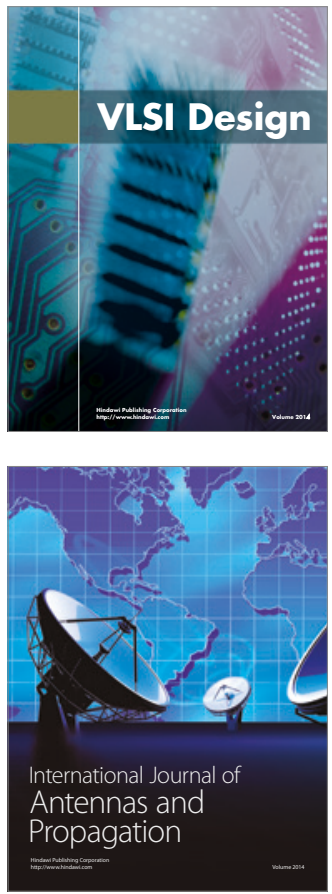

\section{Rotating}

Machinery
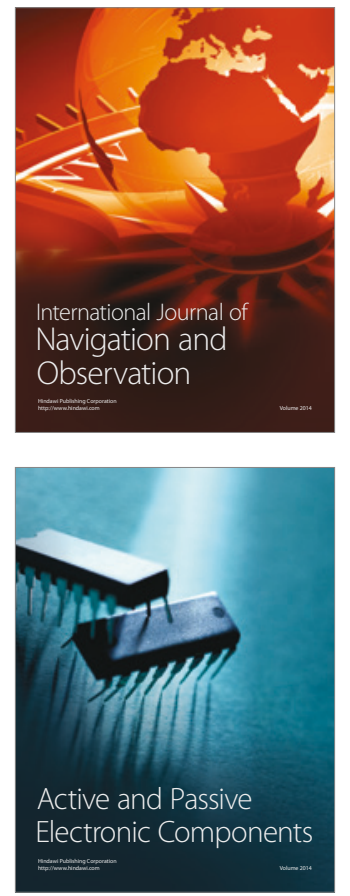
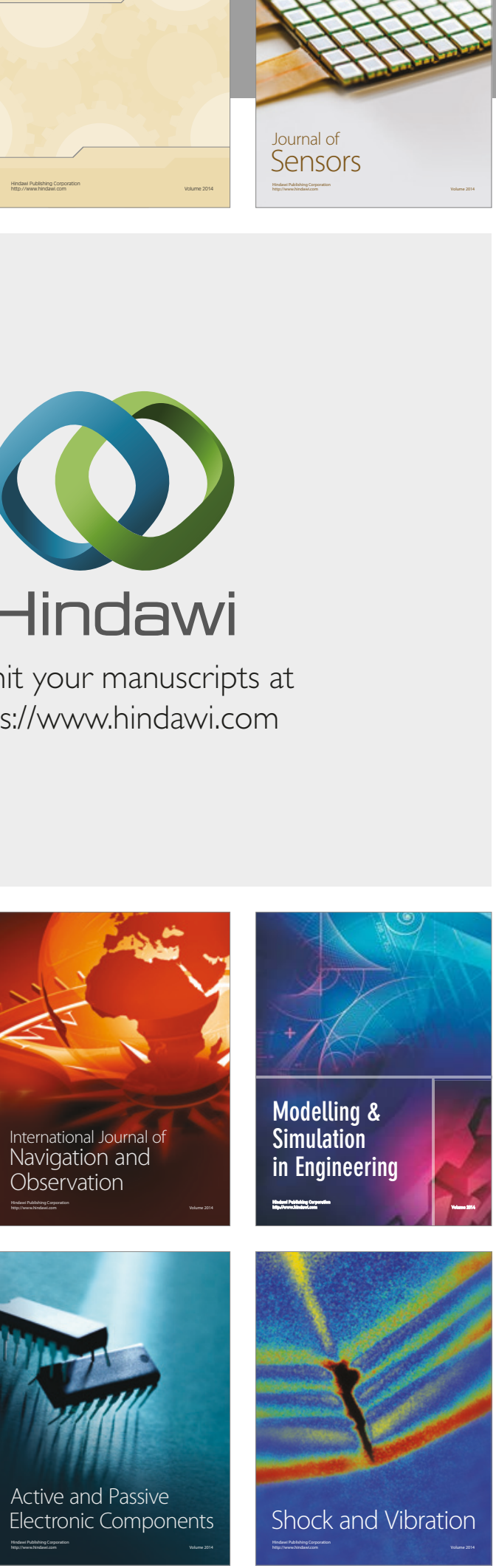
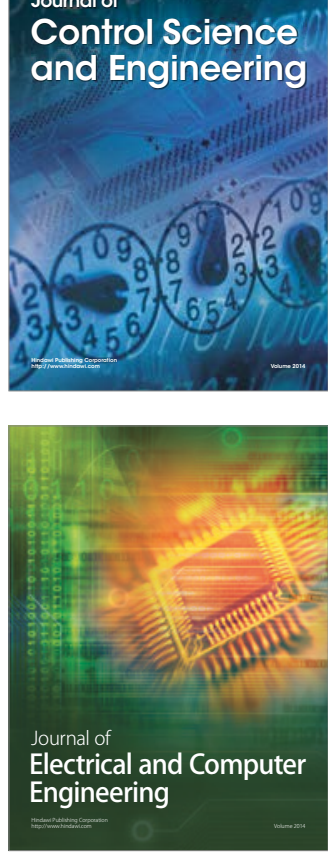

Distributed

Journal of

Control Science

and Engineering
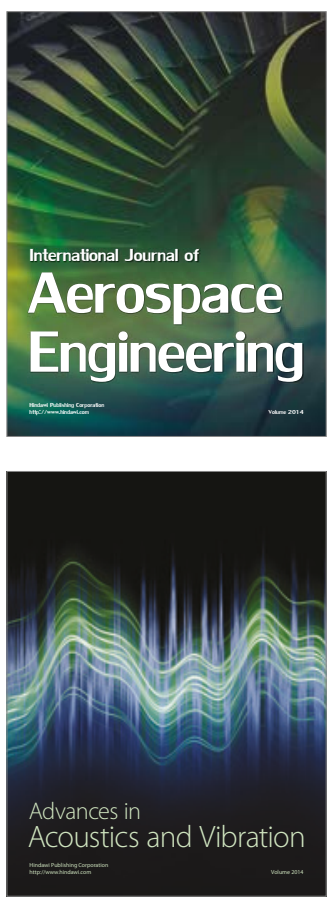

Sensor Networks 